\title{
Resultados a mediano plazo de la prótesis abisagrada rotatoria en el reemplazo total de rodilla primario y de revisión
}

\author{
Sebastián Pereira, ${ }^{\star}$ Fernando Bidolegui," Pablo Maletti," Gabriel Vindver \\ "Servicio de Ortopedia y Traumatología, Hospital Sirio Libanés, ECICARO, Ciudad Autónoma de Buenos Aires, Argentina \\ ${ }^{*}$ Servicio de Ortopedia y Traumatología, Hospital Provincial de Neuquén, Neuquén, Argentina
}

\section{RESUMEN}

Objetivo: Comunicar los resultados clínicos y radiológicos a mediano plazo de 34 artroplastias totales de rodilla abisagradas rotatorias. Materiales y Métodos: Se evaluó a un grupo de 34 prótesis abisagradas de rodilla. Se indicó una bisagra a 10 pacientes $(29,5 \%)$ sometidos a una cirugía primaria y a 24 (70,5\%), a una cirugía de revisión. La edad promedio de la serie era de 78.5 años (rango 54-85). Resultados: El seguimiento promedio fue de 6.5 años (rango 2-12). El rango de movilidad conseguido fue $110^{\circ}$ de flexión (rango $70-130^{\circ}$ ) y $5^{\circ}$ de extensión (rango $0-20^{\circ}$ ). El puntaje promedio en el KSS mejoró de 38 antes de la cirugía a 82 en el posoperatorio. En la evaluación radiológica, se detectaron líneas radiolúcidas $>2 \mathrm{~mm}$ alrededor del componente femoral o tibial, 0 alrededor de los vástagos, en 8 casos (23\%). Cinco de 34 pacientes $(14,7 \%)$ sufrieron complicaciones. En tres casos (8,9\%), se trató de una infección profunda. Dos pacientes (5,9\%) tuvieron complicaciones rotulofemorales. La supervivencia de la prótesis libre de revisión a los 6.5 años fue del 94\%. Si consideramos el aflojamiento aséptico como causa de revisión, la supervivencia fue del $100 \%$. Conclusiones: Las prótesis abisagradas rotatorias de rodilla modernas logran buenos resultados funcionales y de alivio del dolor. También se asocian a bajas tasas de aflojamiento aséptico a mediano plazo. Sin embargo, las complicaciones sépticas son frecuentes.

Palabras clave: Bisagra rotatoria de rodilla; artroplastia de revisión; artroplastia primaria.

Nivel de Evidencia: IV

Mid-term outcomes using rotating-hinge prosthesis for primary and revision total knee arthroplasty

\section{ABSTRACT}

Objective: To analyze the clinical and radiological mid-term outcomes of 34 rotating-hinge total knee arthroplasties. Materials and Methods: We studied 34 hinge knee prostheses. The arthroplasty indication was in the setting of primary surgery for 10 patients $(29.5 \%)$ and in the setting of revision for 24 patients $(70.5 \%)$. The overall average age was 78.5 years (range, $54-85$ years). Results: The average follow-up was 6.5 months (range, 2-12). The achieved range of motion (ROM) was $110^{\circ}$ of flexion (range, $70^{\circ}-130^{\circ}$ ) and $5^{\circ}$ of extension (range, $0^{\circ}-20^{\circ}$ ). The average Knee Society Score (KSS) improved from 38 in the preoperative period to 82 in the postoperative period. Radiolucent lines $(>2 \mathrm{~mm}$ ) around the femoral or tibial components or around the stems were observed in 8 patients $(23 \%)$. Five out of 34 patients $(14.7 \%)$ developed complications. Three patients $(8.9 \%)$ developed a deep infection. Two patients (5.9\%) developed patellofemoral complications. Prosthesis survivorship without revision was $94 \%$ at 6.5 years. And, considering aseptic loosening as a revision cause, prosthesis survivorship was $100 \%$. Conclusion: Modern rotatinghinge knee prostheses provide good outcomes in terms of function and pain relief. They also provide a low rate of aseptic loosening at mid-term follow-up. However, deep infection and septic loosening are common.

Key words: Rotating hinge knee; revision knee arthroplasty; primary knee arthroplasty. Level of Evidence: IV

Recibido el 24-10-2017. Aceptado luego de la evaluación el 23-8-2019 • Dr. SEBASTIÁN PEREIRA • sebopereira@ hotmail.com

Cómo citar este artículo: Pereira S, Bidolegui F, Maletti P, Vindver G. Resultados a mediano plazo de la prótesis abisagrada rotatoria en el reemplazo total de rodilla primario y de revisión. Rev Asoc Argent Ortop Traumatol 2020;85(1):49-55. https://doi.org/10.15417/issn. 1852-7434.2020.85.1.779 


\section{INTRODUCCIÓN}

Uno de los objetivos principales de cualquier artroplastia de rodilla, sea esta primaria o de revisión, es lograr una rodilla estable que permita una articulación funcional y duradera en el tiempo. La presencia de ligamentos colaterales competentes es un requisito a la hora de realizar un reemplazo total de rodilla. No obstante, en los casos de artroplastias primarias con un severo deseje o de una artroplastia de revisión con severo defecto óseo o una severa inestabilidad ligamentaria, suele ser necesario recurrir a implantes constreñidos, como las prótesis abisagradas. Las primeras prótesis abisagradas de rodilla diseñadas en las décadas de 1970 y 1980 estaban asociadas a altos índices de aflojamiento aséptico, ${ }^{1,2} \sin$ embargo, las mejoras en el diseño han disminuido las tasas de falla comunicadas inicialmente..$^{3-11}$ Las bisagras rotatorias actuales proveen una gran estabilidad intrínseca y, al mismo tiempo, permiten movimientos de rotación que replican la biomecánica normal de la rodilla y, de esta manera, disminuyen las fuerzas transmitidas a la interfaz cemento-hueso. ${ }^{6-11}$

El objetivo de este estudio es comunicar los resultados clínicos y radiológicos a mediano plazo de 34 artroplastias totales de rodilla abisagradas rotatorias.

\section{MATERIALES Y MÉTODOS}

Se llevó a cabo un estudio retrospectivo de 36 prótesis abisagradas de rodilla en 35 pacientes no oncológicos, realizadas por dos equipos quirúrgicos, en diferentes centros, entre diciembre de 2003 y enero de 2014. Dos pacientes fueron excluidos, porque se perdieron en el seguimiento; así la serie quedó conformada por 34 prótesis en 33 pacientes. Se indicó una bisagra en 10 casos (29,5\%), en una cirugía primaria y en 24 casos (70,5\%), en una cirugía de revisión. En el grupo de cirugía primaria, las indicaciones fueron: genu valgo severo con insuficiencia del ligamento colateral medial (6 casos), genu varo severo con insuficiencia del ligamento colateral lateral ( 2 casos), recurvatum por secuela de poliomielitis ( 1 caso) y una lesión crónica del ligamento colateral medial sin deseje en un paciente de 78 años. En el grupo de revisiones, la indicación fue una severa insuficiencia ligamentaria, asociada o no a una pérdida ósea. En 14 de ellos (58\%), se indicó en el segundo tiempo de revisión de un aflojamiento séptico y, en los 10 casos restantes, por inestabilidad. La edad promedio de la serie fue de 78.5 años (rango 54-85). Diecinueve (56\%) pacientes eran mujeres y 15 (44\%), hombres. En 19 casos, se trató de la rodilla derecha y, en 15, de la izquierda (Tabla).

Se utilizó la prótesis abisagrada rotatoria total de rodilla Endo-Model® (Link®, Hamburgo, Alemania) en 27 casos y la B-Rotax $®$ (Aston Medical $®$, Francia), en los siete restantes.

Todas las rodillas fueron evaluadas con el Knee Society Score (KSS) antes de la cirugía y en el posoperatorio. ${ }^{12}$ Para medir el rango de movilidad se empleó un goniómetro con el paciente en decúbito dorsal antes de la cirugía y en el posoperatorio. Las radiografías posoperatorias fueron evaluadas buscando signos de aflojamiento. Se administraron $2 \mathrm{~g}$ de cefazolina como profilaxis antibiótica, y $15 \mathrm{mg} / \mathrm{kg}$ de ácido tranexámico antes de la cirugía y una segunda dosis en el momento del cierre. ${ }^{13}$ En ningún caso, utilizamos manguito hemostático. La incisión cutánea se realizó en la línea media, en el caso de las revisiones usamos la incisión previa y, si había más de una, la más lateral. Los seis pacientes con un severo deseje en valgo fueron operados a través de un abordaje pararrotuliano externo. ${ }^{14}$ En el resto de los casos de cirugía primaria, como en todas las revisiones, utilizamos un abordaje pararrotuliano interno. En 18 casos (53\%), usamos un recorte en el tendón del músculo recto anterior o "rectus snip" para facilitar la exposición. En el caso 15, durante una revisión, fue necesario realizar una osteotomía de la tuberosidad anterior de la tibia para facilitar la extracción del vástago tibial (Figura 1). Siempre usamos vástagos cementados y cemento con antibiótico. Solo, en casos seleccionados, implantamos el componente rotuliano según su compromiso degenerativo en las cirugías primarias y el remanente óseo en las revisiones. Como profilaxis antitrombótica, se administraron $40 \mathrm{mg} /$ día de heparina de bajo peso molecular, por vía subcutánea, durante 30 días, a partir de las $12 \mathrm{~h}$ posoperatorias. El protocolo de rehabilitación consistió en ejercicios de movilidad de la rodilla y marcha con andador con carga según tolerancia, a partir de las 48 horas. 
Tabla. Datos demográficos, indicación y resultados

\begin{tabular}{|c|c|c|c|c|c|c|c|c|}
\hline Caso & Edad & Lado & Sexo & Patología & Marca & $\begin{array}{c}\text { KSS } \\
\text { preoperatorio }\end{array}$ & $\begin{array}{c}\text { KSS } \\
\text { posoperatorio }\end{array}$ & Complicaciones \\
\hline 1 & 54 & I & M & Inestabilidad protésica & Link® & 35 & 90 & \\
\hline 2 & 74 & $\mathrm{D}$ & $\mathrm{F}$ & 2do tiempo infección & Link® & 40 & 85 & \\
\hline 3 & 68 & $\mathrm{D}$ & M & 2do tiempo infección & Link® & 45 & 85 & \\
\hline 4 & 77 & $\mathrm{D}$ & $\mathrm{F}$ & 2do tiempo infección & Link® & 37 & 93 & \\
\hline 5 & 72 & I & $\mathrm{F}$ & 2do tiempo infección & Link® & 30 & 75 & \\
\hline 6 & 82 & I & M & Inestabilidad protésica & Link® & 45 & 90 & \\
\hline 7 & 79 & $\mathrm{D}$ & $\mathrm{F}$ & Inestabilidad protésica & Link® & 30 & 81 & \\
\hline 8 & 67 & $\mathrm{D}$ & M & Genu valgo severo & Link® & 41 & 57 & \\
\hline 9 & 79 & I & M & Genu varo severo & Link® & 34 & 85 & \\
\hline 10 & 72 & $\mathrm{D}$ & $\mathrm{F}$ & Genu varo severo & $\operatorname{Rotax} ®$ & 42 & 91 & \\
\hline 11 & 72 & $\mathrm{D}$ & M & 2do tiempo infección & $\operatorname{Rotax} \AA$ & 47 & 83 & \\
\hline 12 & 77 & I & $\mathrm{F}$ & Genu valgo severo & $\operatorname{Rotax} ®$ & 35 & 76 & \\
\hline 13 & 79 & $\mathrm{D}$ & $\mathrm{F}$ & Genu valgo severo & $\operatorname{Rotax}{ }^{\circledR}$ & 30 & 82 & Infección \\
\hline 14 & 69 & I & M & 2do tiempo infección & Link® & 43 & 81 & \\
\hline 15 & 76 & $\mathrm{D}$ & $\mathrm{F}$ & 2do tiempo infección & Link® & 36 & 92 & Infección \\
\hline 16 & 80 & I & $\mathrm{F}$ & Inestabilidad protésica & Link® & 39 & 90 & \\
\hline 17 & 85 & $\mathrm{D}$ & M & Genu valgo severo & $\operatorname{Rotax}{ }^{\circledR}$ & 43 & 75 & Luxación rotuliana \\
\hline 18 & 68 & I & $\mathrm{F}$ & 2do tiempo infección & $\operatorname{Rotax}{ }^{\circledR}$ & 40 & 91 & \\
\hline 19 & 62 & D & M & Recurvatum & Link® & 30 & 82 & \\
\hline 20 & 78 & I & $\mathrm{F}$ & 2do tiempo infección & Link® & 30 & 85 & \\
\hline 21 & 71 & $\mathrm{D}$ & $\mathrm{F}$ & 2do tiempo infección & Link® & 5 & 50 & Infección \\
\hline 22 & 65 & I & M & Inestabilidad protésica & Link® & 40 & 85 & \\
\hline 23 & 67 & $\mathrm{D}$ & $\mathrm{F}$ & Inestabilidad protésica & Link® & 20 & 92 & \\
\hline 24 & 75 & $\mathrm{D}$ & $\mathrm{F}$ & Inestabilidad protésica & Link® & 35 & 87 & \\
\hline 25 & 73 & I & M & Inestabilidad protésica & Link® & 30 & 69 & Luxación rotuliana \\
\hline 26 & 85 & $\mathrm{D}$ & $\mathrm{F}$ & Genu valgo severo & Link® & 41 & 87 & \\
\hline 27 & 53 & I & $\mathrm{F}$ & Inestabilidad protésica & Link® & 37 & 87 & \\
\hline 28 & 65 & $\mathrm{D}$ & M & Inestabilidad protésica & Link® & 45 & 93 & \\
\hline 29 & 70 & $\mathrm{D}$ & M & 2do tiempo infección & $\operatorname{Rotax}{ }^{\circledR}$ & 45 & 69 & \\
\hline 30 & 70 & I & $\mathrm{F}$ & 2do tiempo infección & Link® & 30 & 91 & \\
\hline 31 & 72 & I & $\mathrm{F}$ & Genu valgo severo & Link® & 15 & 82 & \\
\hline 32 & 68 & $\mathrm{D}$ & M & 2do tiempo infección & Link® & 45 & 93 & \\
\hline 33 & 78 & $\mathrm{D}$ & M & Lesión crónica de LCM & Link® & 40 & 70 & \\
\hline 34 & 83 & I & $\mathrm{F}$ & 2do tiempo infección & Link® & 34 & 85 & \\
\hline
\end{tabular}

$\mathrm{I}=$ izquierdo, $\mathrm{D}=$ derecho, $\mathrm{F}=$ femenino, $\mathrm{M}=$ masculino, $\mathrm{LCM}=$ ligamento colateral medial, $\mathrm{KSS}=$ Knee Society Score . 


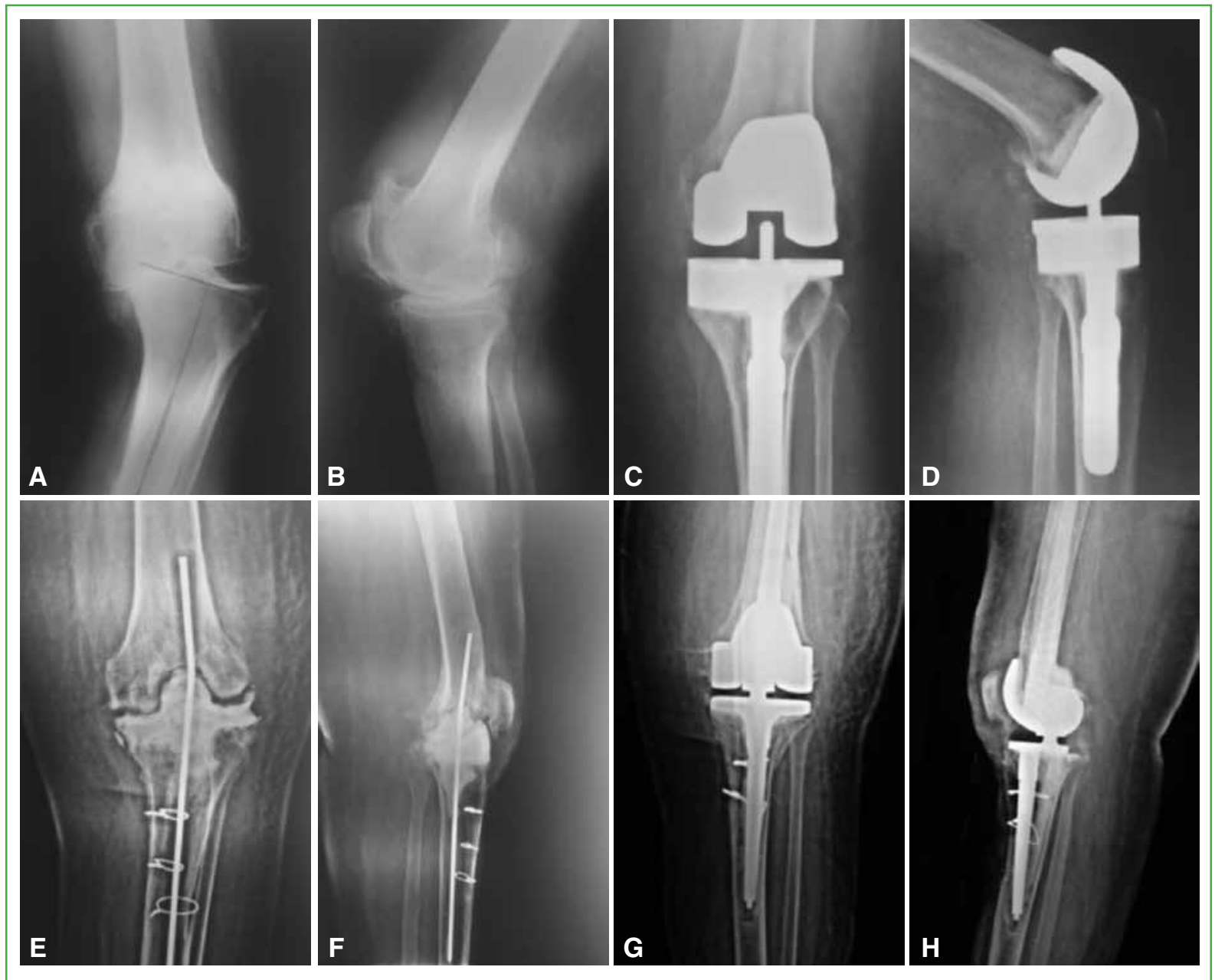

Figura 1. Caso 15. A y B. Genu varo severo. C y D. Artroplastia total de rodilla con aumento tibial medial y vástago no cementado. E y F. Primer tiempo de revisión por aflojamiento séptico. Osteotomía de la tuberosidad anterior de la tibia y colocación de un espaciador no articulado. G y H. Segundo tiempo de revisión con una prótesis abisagrada total de rodilla.

\section{RESULTADOS}

El seguimiento promedio fue de 6.5 años (rango 2-12). El rango de movilidad conseguido fue $110^{\circ}$ de flexión (rango $70-130^{\circ}$ ) y $5^{\circ}$ de extensión (rango $0-20^{\circ}$ ). El eje femorotibial fue restaurado en todos los casos. El puntaje promedio del KSS mejoró de 38 antes de la cirugía a 82 en el posoperatorio. El resultado fue considerado bueno (KSS $\geq 80)$ en 26 casos $(76,5 \%)$, regular en seis casos $(17,6 \%)$ y pobre en dos casos $(5,9 \%)$. En la evaluación radiológica, se detectaron líneas radiolúcidas $>2 \mathrm{~mm}$ alrededor del componente femoral o tibial, o alrededor de los vástagos, en ocho pacientes (23\%). En un caso, estas fueron progresivas y correspondieron al paciente con aflojamiento séptico, que requirió una revisión.

Se detectaron complicaciones en cinco de 34 prótesis $(14,7 \%)$. En tres pacientes $(8,9 \%)$, se trató de una infección profunda, todas reinfecciones. Dos de los tres casos fueron tratados con limpiezas quirúrgicas y tratamiento antibiótico supresor (casos 13 y 15). El restante requirió la extracción de la prótesis y la colocación de un nuevo espaciador; sin embargo, el paciente rechazó una nueva cirugía (caso 21). Dos pacientes (5,9\%) tuvieron complicaciones rotulofemorales; uno requirió, debido a reiterados episodios de luxación rotuliana, una revisión en la cual se implantó el componente rotuliano y se liberó el retináculo lateral (caso 17) (Figura 2). El paciente restante presentaba episodios de subluxación rotuliana y, como rechazó la cirugía, fue tratado, en forma conservadora, con un plan de fortalecimiento muscular (caso 31). 

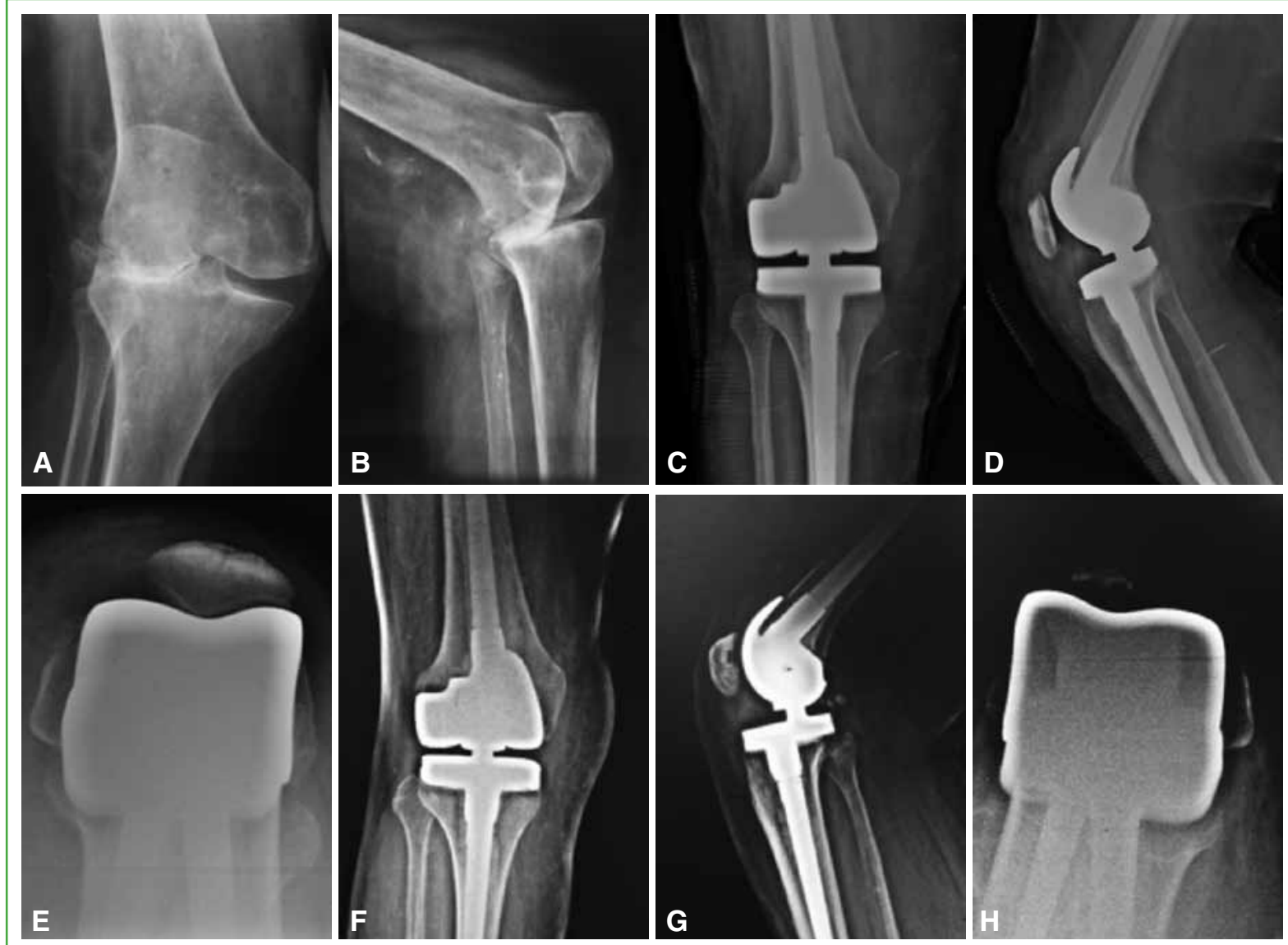

Figura 2. Caso 17. A y B. Paciente con genu valgo severo. C-E. Radiografías posoperatorias donde se evidencia una leve lateralización de la rótula. F-H. Ante los síntomas por la inestabilidad rotuliana, se liberó el retináculo lateral y se implantó el componente rotuliano.

La supervivencia de la prótesis libre de revisión a los 6.5 años fue del 94\%. Si consideramos el aflojamiento aséptico como causa de revisión, la supervivencia fue del $100 \%$.

\section{DISCUSIÓN}

Las prótesis abisagradas de rodilla se han utilizado para el tratamiento de la inestabilidad ligamentaria severa en el escenario de cirugías de revisión y aun en casos seleccionados de artroplastias primarias. Los primeros diseños datan de la década de 1950 y correspondían a verdaderas bisagras que solo permitían movimientos de flexión y extensión. Si bien la estabilidad inicial era excelente, la imposibilidad de estas prótesis de permitir una movilidad más fisiológica de la rodilla exponía al implante a una alta solicitación mecánica, la cual era transmitida a la interfaz hueso-cemento-implante que daba como resultado altas tasas de aflojamiento mecánico. ${ }^{1-3}$

En la década de 1970, se diseñaron las primeras bisagras rotatorias con el intento de disminuir las complicaciones asociadas a las bisagras fijas. Sin embargo, los resultados iniciales a corto y mediano plazo no fueron los esperados. ${ }^{1-3}$ Finalmente, a partir de la década de 1990, de la mano de la tercera generación de prótesis abisagradas, se lograron resultados más alentadores. En 2014, Gehrke y cols., en su serie de 238 bisagras en casos primarios, informaron una tasa de revisión por aflojamiento aséptico del 0,5\% a los 13 años de seguimiento. Estos autores aclaran, sin embargo, que todos los casos de la serie fueron reemplazos primarios y esto podría justificar este bajo porcentaje. ${ }^{15}$ En 2017, Cottino y cols. reportaron una tasa de revisión por aflojamiento aséptico a los 10 años del $4,5 \%$ en su serie de 408 prótesis abisagradas. ${ }^{16}$ Estos resultados resaltan el significativo descenso en las fuerzas transmitidas hacia la interfaz hueso-cemento-implante con los implantes modernos respecto a los primeros diseños. 
Las modificaciones de los diseños más modernos también han mejorado la congruencia rotulofemoral no resuelta inicialmente por las primeras prótesis abisagradas y responsable de las complicaciones rotulianas. En una serie de 38 pacientes, con un seguimiento de 50 meses, Rand y cols. comunicaron un $22 \%$ de complicaciones rotulofemorales. ${ }^{1}$ En 2001, Springer y cols. reportaron un $13 \%$ de complicaciones rotulianas en una serie de 188 pacientes. ${ }^{17}$ Cottino y cols. detectaron un $1 \%$ de complicaciones rotulofemorales con las bisagras modernas en 408 casos. ${ }^{16}$ En nuestra serie, dos pacientes $(5,9 \%)$ presentaron complicaciones rotulianas, y uno de ellos fue sometido a una revisión.

La infección sigue siendo una de las complicaciones más frecuentes. La necesidad de una gran exposición y liberación de partes blandas, así como el prolongado tiempo quirúrgico y las múltiples comorbilidades serían responsables de la alta tasa de infecciones comunicada. Springer y cols., en su serie de prótesis abisagradas de rodilla en pacientes sin neoplasias, detectaron una infección en cinco (19\%) de los 26 pacientes. ${ }^{18}$ En la serie de Cottino y cols., la tasa de revisión a 10 años asciende al 22,5\% cuando incluyen las fallas por motivos sépticos. Sin embargo, destacan que estos resultados no sorprenden, ya que la mayoría de los pacientes sufre varias comorbilidades y muchos de ellos tienen múltiples cirugías previas, la mayoría por infección. ${ }^{16}$ Gehrke y cols. comunicaron un $2 \%$ de infección en su serie de 238 bisagras primarias. ${ }^{15}$ En nuestra serie, la tasa de infección del 8,9\% se ubica en el medio de los resultados publicados. Esto se puede explicar, porque incluimos tanto casos primarios como revisiones.

Los resultados funcionales y las mejoras en el rango de movilidad en las distintas series han sido alentadores. Nuestros resultados son consistentes con diferentes reportes. En 2010, Hossain y cols. comunicaron, en un estudio comparativo de pacientes sometidos a una artroplastia de rodilla, similares resultados de satisfacción y rango de movilidad posoperatorios, independientemente del tipo de prótesis, estabilizada posterior, constreñida o abisagrada. ${ }^{4}$

\section{CONCLUSIONES}

Las prótesis abisagradas rotatorias de rodilla modernas logran buenos resultados funcionales y de alivio del dolor, inclusive en los escenarios de cirugías de revisión más complejos. También se asocian a bajas tasas de aflojamiento aséptico a mediano plazo. Sin embargo, las complicaciones sépticas asociadas siguen siendo las responsables del mayor número de falla, debido, en parte, a las comorbilidades de los pacientes y a la frecuente presencia de infección previa.

Conflicto de intereses: Los autores no declaran conflictos de intereses.

\section{BIBLIOGRAFÍA}

1. Rand JA, Chao EY, Stauffer RN. Kinematic rotating-hinge total knee arthroplasty. J Bone Joint Surg Am 1987;69(4): 489-97. PMID: 3571306

2. Duquennoy A, Decoulx J, Epinette JA, Letendart J. [Hinge prosthesis of the knee. A propos of 185 cases]. Rev Chir Orthop Reparatrice Appar Mot 1983;69(6):465-74. PMID: 6227949

3. Pradhan NR, Bale L, Kay P, Porter ML Salvage revision total knee replacement using the Endo-Model rotating hinge prosthesis. Knee 2004;11(6):469-73. https://doi.org/10.1016/j.knee.2004.03.001

4. Hossain F, Patel S, Haddad FS. Midterm assessment of causes and results of revision total knee arthroplasty. Clin Orthop Relat Res 2010;468(5):1221-8. https://doi.org/10.1007/s11999-009-1204-0

5. Barrack RL. Evolution of the rotating hinge for complex total knee arthroplasty. Clin Orthop Relat Res 2001;(392): 292-9. https://doi.org/10.1097/00003086-200111000-00038 
6. Joshi N, Navarro-Quilis A. Is there a place for rotating-hinge arthroplasty in knee revision surgery for aseptic loosening? J Arthroplasty 2008;23(8):1204-11. https://doi.org/10.1016/j.arth.2007.10.01 10.1016/j.arth.2007.10.01

7. Rodríguez-Merchán EC, Gómez-Cardero P, Martínez-Lloreda Á. Revision knee arthroplasty with a rotating-hinge design in elderly patients with instability following total knee arthroplasty. J Clin Orthop Trauma 2015;6(1):19-23. https://doi.org/10.1016/j.jcot.2014.11.001

8. Hernández-Vaquero D, Sandoval-García MA. Hinged total knee arthroplasty in the presence of ligamentous deficiency. Clin Orthop Relat Res 2010;468(5):1248-53. https://doi.org/10.1007/s11999-009-1226-7

9. Westrich GH, Mollano AV, Sculco TP, Buly RL, Laskin RS, Windsor R. Rotating hinge total knee arthroplasty in severely affected knees. Clin Orthop Relat Res 2000;(379):195-208. https://doi.org/10.1097/00003086-20001000000023

10. Pour AE, Parvizi J, Slenker N, Purtill JJ, Sharkey PF. Rotating hinged total knee replacement: use with caution. $J$ Bone Joint Surg Am 2007;89(8):1735-41. https://doi.org/10.2106/JBJS.F.00893

11. Guenoun B, Latargez L, Frelson M, Defossez G, Salas N, Gayet L.-E. Complication following rotating hinge Endo-Model (Link) knee arthroplasty. Orthop Traumatol Surg Res 2009;95(7):529-36. https://doi.org/10.1016/j. otsr.2009.07.013

12. Insall JN, Dorr LD, Scott RD, Scott WN. Rationale of the Knee Society clinical rating system. Clin Orthop Relat Res 1989;(248):13-4. PMID: 2805470

13. Bidolegui F, Arce G, Lugones A, Pereira S, Vindver G. Tranexamic acid reduces blood loss and transfusion in patients undergoing total knee arthroplasty without tourniquet: a prospective randomized controlled trial. Open Orthop J 2014;8:250-4. https://doi.org/10.2174/1874325001408010250

14. Keblish PA. The lateral approach to the valgus knee. Surgical technique and analysis of 53 cases with over two-year follow-up evaluation. Clin Orthop Relat Res 1991;(271):52-62. PMID: 1914314

15. Gehrke T, Kendoff D, Haasper C. Management factorials in total knee arthroplasty. The role of hinges in primary total knee replacement. Bone Joint J Br 2014;96(11 Suppl A):93-5. https://doi.org/10.1302/0301-620X.96B11.34143

16. Cottino U, Abdel MP, Perry KI, Mara KC, Lewallen DG, Hanssen AD. Long-term results after total knee arthroplasty with contemporary rotating-hinge prostheses. Bone Joint Surg Am 2017;99(4):324-30. https://doi. org/10.2106/JBJS.16.00307

17. Springer BD, Hanssen AD, Sim FH, Lewallen DG. The kinematic rotating hinge prosthesis for complex knee arthroplasty. Clin Orthop Relat Res 2001;(392):283-91. https://doi.org/10.1097/00003086-200111000-00037

18. Springer BD, Sim FH, Hanssen AD, Lewallen DG. The modular segmental kinematic rotating hinge for nonneoplastic limb salvage. Clin Orthop Relat Res 2004;(421):181-7. https://doi.org/10.1097/01. blo.0000126306.87452.59 